\title{
COMPOSITION OPERATORS BELONGING TO SCHATTEN CLASS $\mathcal{S}_{p}$
}

\author{
CHENG YUAN and ZE-HUA ZHOU ${ }^{凶}$
}

(Received 17 July 2009)

\begin{abstract}
We investigate the composition operators $C_{\varphi}$ acting on the Bergman space of the unit disc $D$, where $\varphi$ is a holomorphic self-map of $D$. Some new conditions for $C_{\varphi}$ to belong to the Schatten class $\mathcal{S}_{p}$ are obtained. We also construct a compact composition operator which does not belong to any Schatten class.
\end{abstract}

2000 Mathematics subject classification: primary 47B38; secondary 46E15, 32A37.

Keywords and phrases: Bergman spaces, composition operators, Schatten class.

\section{Introduction}

Let $L_{a}^{2}(D)$ be the Bergman space of the unit disc $D$ in $\mathbb{C}$. Recall that an analytic function $f \in L_{a}^{2}(D)$ if and only if

$$
\|f\|^{2}=\int_{D}|f(z)|^{2} d A(z)<\infty,
$$

where $d A(z)=(1 / \pi) d x d y$ is the normalized area measure on the unit disk. The space $L_{a}^{2}(D)$, when equipped with the obvious inner product, is a Hilbert space with reproducing kernel $K(z, w)=(1-z \bar{w})^{-2}$. For a holomorphic map $\varphi: D \rightarrow D$, the composition operator $C_{\varphi}$ with the symbol $\varphi$ on $L_{a}^{2}(D)$ is defined by $C_{\varphi}(f)=f \circ \varphi$. It is well known that $C_{\varphi}$ is always bounded on $L_{a}^{2}(D)[1,5]$.

Recall that, for any $1 \leq p<\infty$, the Schatten class $\mathcal{S}_{p}$ on $L_{a}^{2}(D)$ consists of linear operators $T$ satisfying $\left\{\operatorname{tr}\left(|T|^{p}\right)\right\}^{1 / p}=\left\{\operatorname{tr}\left(\left(T^{*} T\right)^{p / 2}\right)\right\}^{1 / p}<\infty$, where $\operatorname{tr}(T)$ is the trace of $T$ defined by

$$
\operatorname{tr}(T)=\sum_{n=1}^{\infty}\left\langle T e_{n}, e_{n}\right\rangle .
$$

To characterize the Schatten class composition operators, it is usual to consider the integral

$$
\int_{D}\left(\frac{1-|z|^{2}}{1-|\varphi(z)|^{2}}\right)^{p} d \lambda(z)<\infty,
$$

Supported in part by the National Natural Science Foundation of China (grants 10971153, 10671141).

(c) 2010 Australian Mathematical Publishing Association Inc. 0004-9727/2010 \$16.00 
where

$$
d \lambda(z)=\left(1-|z|^{2}\right)^{-2} d A(z)
$$

is the Möbius invariant measure on $D$. It is well known that (1.1) is sufficient for $C_{\varphi} \in \mathcal{S}_{p}$ when $1<p \leq 2$ and necessary for $C_{\varphi} \in \mathcal{S}_{p}$ when $2 \leq p<\infty$ (see [4], for example). Recently, Zhu characterized the Schatten class composition operators on the weighted Bergman space when $2 \leq p<\infty$. He proved that when $\varphi$ has bounded valence, that is, there is a positive integer $N$ such that for every $z \in D$ the set $\varphi^{-1}(z)$ contains at most $N$ points, $C_{\varphi} \in \mathcal{S}_{p}$ if and only if (1.1) holds (see [8]). Later, Xia constructed an analytic function $\varphi: D \rightarrow D$ such that (1.1) holds, but $C_{\varphi} \notin \mathcal{S}_{p}$ for any $2<p<\infty$ (see [6]). The Schatten class weighted composition operators on Bergman spaces were characterized in [2]. For various $p$, composition operators belonging to different $\mathcal{S}_{p}$ Hardy space were constructed in [3]. Motivated by these works, we characterize the composition operators belonging to the Schatten class $\mathcal{S}_{p}$ with different conditions. We give a new condition for $C_{\varphi} \in \mathcal{S}_{p}$ and construct a compact composition operator which is not in any Schatten class.

This paper is organized as follows. In Section 2 we analyze composition operators and give some conditions for $C_{\varphi} \in \mathcal{S}_{p}$ for various $p$. The example $C_{\varphi} \in \mathcal{S}_{p}$ for which (1.1) fails is given in Section 3. The main idea is inspired by [6, 9].

The notation $U \approx V$ means that there are two constants $c_{1}$ and $c_{2}$ independent of $U$ and $V$, the implied variables or functions, such that $c_{1} V \leq U \leq c_{2} V$. The condition $U \leq c_{2} V$ will simply be written as $U \preceq V$ or $V \succeq U$.

\section{Relation to Toeplitz operators on Bergman spaces}

Composition operators are closely related to Toeplitz operators on Bergman spaces, and this connection has been used to characterize Schatten class composition operators (see [4, 8], for example).

We recall that the Toeplitz operator $T_{\mu}$ induced by a finite positive Borel measure on $D$ is densely defined on $L_{a}^{2}(D)$ by

$$
T_{\mu} f(z)=\int_{D} K(z, w) f(w) d \mu(w) .
$$

The Berezin symbol $\tilde{\mu}$ of $\mu$ is given as follows:

$$
\tilde{\mu}(z)=\int_{D} \frac{\left(1-|z|^{2}\right)^{2}}{|1-z \bar{w}|^{4}} d \mu(w), \quad z \in D .
$$

We will need the following results from [8].

Lemma 2.1 [8, Lemma 2.1]. Suppose that $0<p<+\infty$ and $\mu$ is a finite position Borel measure on D. Then $T_{\mu}$ is in $\mathcal{S}_{p}$ of $L_{a}^{2}(D)$ if and only if $\tilde{\mu}$ is in $L^{p}(D, d \lambda)$.

Lemma 2.2 [8, Lemma 2.2]. Suppose that $\varphi: D \rightarrow D$ is analytic and $C_{\varphi}$ is the composition operator on $L_{a}^{2}(D)$. Then $C_{\varphi}^{*} C_{\varphi}=T_{\mu}$, where $\mu=A \circ \varphi^{-1}$ is the pullback measure of $A$ induced by $\varphi$. 
Now we consider $C_{\varphi}^{*} C_{\varphi}$ as a Toeplitz operator. Then $C_{\varphi} \in \mathcal{S}_{p}$ if and only if $C_{\varphi}^{*} C_{\varphi} \in \mathcal{S}_{p / 2}$ if and only if $T_{\mu} \in \mathcal{S}_{p / 2}$, where $\mu$ is given in Lemma 2.2 above. Using Lemma 2.1, $T_{\mu} \in \mathcal{S}_{p / 2}$ if and only if $\tilde{\mu} \in L^{p / 2}(D, d \lambda)$. That is,

$$
\begin{aligned}
\tilde{\mu}(z) & =\int_{D} \frac{\left(1-|z|^{2}\right)^{2}}{|1-z \bar{w}|^{4}} d A \circ \varphi^{-1}(w) \\
& =\int_{D} \frac{\left(1-|z|^{2}\right)^{2}}{|1-z \overline{\varphi(w)}|^{4}} d A(w) \in L^{p / 2}(D, d \lambda),
\end{aligned}
$$

or equivalently,

$$
\int_{D}\left|\int_{D} \frac{\left(1-|z|^{2}\right)^{2}}{|1-z \overline{\varphi(w)}|^{4}} d A(w)\right|^{p / 2} d \lambda(z)<\infty
$$

If $1<p<2$, or $1 / 2<p / 2<1$, Hölder's inequality and Fubini's theorem imply that

$$
\begin{aligned}
& \int_{D}\left|\int_{D} \frac{\left(1-|z|^{2}\right)^{2}}{|1-z \overline{\varphi(w)}|^{4}} d A(w)\right|^{p / 2} d \lambda(z) \\
& =\int_{D}\left|\int_{D} \frac{\left(1-|z|^{2}\right)^{2}}{|1-z \overline{\varphi(w)}|^{4}} d A(w)\right|^{p / 2} \frac{d A(z)}{\left(1-|z|^{2}\right)^{2}} \\
& \geq \int_{D} \int_{D} \frac{\left(1-|z|^{2}\right)^{p-2}}{|1-z \overline{\varphi(w)}|^{2 p}} d A(w) d A(z) \\
& =\int_{D} \int_{D} \frac{\left(1-|z|^{2}\right)^{p-2}}{|1-z \overline{\varphi(w)}|^{2+p-2+p}} d A(z) d A(w) .
\end{aligned}
$$

Since $p-2>-1$, using the estimate given in [7, p. 53],

$$
\int_{D} \frac{\left(1-|z|^{2}\right)^{p-2}}{|1-z \overline{\varphi(w)}|^{2+p-2+p}} d A(z) \geq \frac{C}{\left(1-|\varphi(w)|^{2}\right)^{p}}
$$

for some absolute constant $C$. This means that

$$
\int_{D} \frac{1}{\left(1-|\varphi(w)|^{2}\right)^{p}} d A(w)<+\infty .
$$

If $p>2$, it follows from Hölder's inequality and Fubini's theorem that

$$
\int_{D}\left|\int_{D} \frac{\left(1-|z|^{2}\right)^{2}}{|1-z \overline{\varphi(w)}|^{4}} d A(w)\right|^{p / 2} d \lambda(z) \leq C \int_{D} \frac{1}{\left(1-|\varphi(w)|^{2}\right)^{p}} d A(w) .
$$

The above argument leads to our next theorem. 
THEOREM 2.3. The condition

$$
\int_{D} \frac{1}{\left(1-|\varphi(w)|^{2}\right)^{p}} d A(w)<+\infty
$$

is necessary for $C_{\varphi} \in \mathcal{S}_{p}$ when $1<p<2$ and sufficient for $C_{\varphi} \in \mathcal{S}_{p}$ when $2<p<\infty$.

Notice that when $p>2,2-4 / p>0$, we can use Hölder's inequality, Fubini's theorem and the estimate in $[7$, p. 53] to get

$$
\begin{aligned}
\int_{D} \mid & \left.\int_{D} \frac{\left(1-|z|^{2}\right)^{2}}{|1-z \overline{\varphi(w)}|^{4}} d A(w)\right|^{p / 2} d \lambda(z) \\
& =\int_{D} \frac{\left|\int_{D} \frac{\left(1-|z|^{2}\right)^{2}}{|1-z \overline{\varphi(w)}|^{4}} d A(w)\right|^{p / 2}}{\left(\left(1-|z|^{2}\right)^{4 / p}\right)^{p / 2}} d A(z) \\
& \geq\left(\int_{D} \int_{D} \frac{\left(1-|z|^{2}\right)^{2}}{|1-z \overline{\varphi(w)}|^{4}} d A(w) \frac{d A(z)}{\left(1-|z|^{2}\right)^{4 / p}}\right)^{p / 2} \\
& =\left(\int_{D} \int_{D} \frac{\left(1-|z|^{2}\right)^{2-4 / p}}{|1-z \overline{\varphi(w)}|^{4}} d A(z) d A(w)\right)^{p / 2} \\
& \approx\left(\int_{D} \frac{1}{\left(1-|\varphi(w)|^{2}\right)^{4 / p}} d A(w)\right)^{p / 2} .
\end{aligned}
$$

Similarly, when $p<2$ and $2-4 / p>-1$, or equivalently $4 / 3<p<2$,

$$
\int_{D}\left|\int_{D} \frac{\left(1-|z|^{2}\right)^{2}}{|1-z \overline{\varphi(w)}|^{4}} d A(w)\right|^{p / 2} d \lambda(z) \preceq\left(\int_{D} \frac{1}{\left(1-|\varphi(w)|^{2}\right)^{4 / p}} d A(w)\right)^{p / 2} .
$$

Indeed, we have the following theorem.

THEOREM 2.4. The condition

$$
\int_{D} \frac{1}{\left(1-|\varphi(w)|^{2}\right)^{4 / p}} d A(w)<+\infty
$$

is sufficient for $C_{\varphi} \in \mathcal{S}_{p}$ when $4 / 3<p<2$ and necessary for $C_{\varphi} \in \mathcal{S}_{p}$ when $2<p<\infty$.

\section{An example}

In this section, we construct a compact composition operator, which does not belong to any Schatten class, by a modification of the construction in [6].

We first let $f(n)$ be a positive decreasing function of $n$ satisfying $f(n) \leq 2 f(n+1)$ and $\sum_{n=1}^{\infty} f(n)=1 / 2$. We define $a_{0}=1 / 2, a_{n}=1 / 2-\sum_{i=1}^{n} f(i)$. Next consider the intervals $J_{n}=\left(a_{n}, a_{n-1}\right)$ and let $I_{n}$ be the left half of $J_{n}$. Then we have that $I_{n}=\left(a_{n},\left(a_{n}+a_{n-1}\right) / 2\right)$. If $\left|I_{n}\right|$ denotes the length of $I_{n}$, then $\left|I_{n}\right|=f(n) / 2$. Define $U=\bigcup_{n=1}^{\infty} I_{n}$. An easy computation gives the following lemma. 
LEMMA 3.1. For any $x \in \mathbb{R} \backslash U$ and $0<a<+\infty$,

$$
m((x-a, x+a) \backslash U) \geq a / 2
$$

where $m$ is the standard Lebesgue measure on $\mathbb{R}$.

We now define a measurable function $u$ on the unit circle $T=\{\tau \in \mathbb{C}:|\tau|=1\}$ as follows:

$$
u\left(e^{i t}\right)= \begin{cases}x(n) & \text { if } t \in I_{n}, n \geq 1, \\ 1 & \text { if } t \in(-\pi, \pi] \backslash U\end{cases}
$$

where $x(n)$ is a positive decreasing function of $n$ with $\lim _{n \rightarrow \infty} x(n)=0$ and $x(n)<1$ for all $n$.

The harmonic extension of $u$ to $D$ will be denoted by the same symbol. We now define

$$
\begin{gathered}
h(z)=\frac{1}{2 \pi} \int_{-\pi}^{\pi} \frac{e^{i t}+z}{e^{i t}-z} u\left(e^{i t}\right) d t, \\
\varphi(z)=\exp (-h(z)), \quad z \in D .
\end{gathered}
$$

It is easy to check that $\Re\{h(z)\}=u(z)>0$ for every $z \in D$. Therefore $\varphi(D) \subset D$. We will analyze different $\varphi$ for different $f(n)$ and $x(n)$.

If we let $\delta=\inf _{0<x \leq 1} x^{-1}\left(1-e^{-x}\right)$ and $\sigma=\sup _{0<x \leq 1} x^{-1}\left(1-e^{-x}\right)$, then we have $1-e^{-x} \geq x \delta$ and $1-e^{-x} \leq x \sigma$. Thus for every $E \subset D$, when $z \in E$,

$$
(1-|\varphi(z)|) \geq \delta u(z) \quad \text { and } \quad(1-|\varphi(z)|) \leq \sigma u(z) .
$$

We also need the following estimate of the Poisson kernel which is given in [6]. For $P(z, \tau)=\left(1-|z|^{2}\right) /|1-z \bar{\tau}|^{2}, \tau \in T$ and $z \in D$, if $1 / 2 \leq r<1$ and $|\theta-t| \leq 5$, then

$$
\frac{\alpha(1-r)}{(1-r)^{2}+(\theta-t)^{2}} \leq \frac{1}{2 \pi} P\left(r e^{i \theta}, e^{i t}\right) \leq \frac{\beta(1-r)}{(1-r)^{2}+(\theta-t)^{2}}
$$

for some absolute constants $0<\alpha<\beta<\infty$.

Using this estimate, we obtain the following lemma.

LEMMA 3.2. For $n \in \mathbb{N}$, let $\rho_{n}=\left|I_{n}\right|$, define

$$
G_{n}=\left\{r e^{i \theta}: \theta \in I_{n}, 0<1-r<\rho_{n}\right\} .
$$

Then there exists a constant $c$ such that

$$
\sup _{z \in G_{n}}|\varphi(z)| \leq e^{-c x(n+1)} .
$$


PRoOF. Since $\theta \in I_{n}, u\left(e^{i t}\right) \geq x(n+1)$ for $\theta \in J_{n} \cup J_{n+1}$. Writing $z=r e^{i \theta}$ with $\theta \in I_{n}$ and $0<r<1-\rho$, then

$$
\begin{aligned}
u\left(r e^{i \theta}\right) & \geq x(n+1) \int_{\theta-\rho_{n}}^{\theta+\rho_{n}} P\left(r e^{i \theta}, e^{i t}\right) d t \\
& \geq x(n+1) \alpha \int_{\theta-\rho_{n}}^{\theta+\rho_{n}} \frac{1-r}{(1-r)^{2}+(t-\theta)^{2}} d t \\
& =x(n+1) \alpha \int_{-\rho_{n}}^{\rho_{n}} \frac{1-r}{(1-r)^{2}+s^{2}} d s \\
& \geq x(n+1) \alpha \int_{-1}^{1} \frac{1}{1+x^{2}} d x
\end{aligned}
$$

where the last inequality is due to the fact that $1-r \leq \rho_{n}$ when $z \in G_{n}$.

Therefore there exists a constant $c$ such that $u\left(r e^{i \theta}\right) \geq c x(n+1)$ for $r e^{i \theta} \in G_{n}$. So $|\varphi(z)|=e^{-u(z)} \leq e^{-c x(n+1)}$ for $z \in G_{n}$.

LEMMA 3.3. There is a constant $C_{1}>0$ such that $u(z) \geq C_{1}$ for every $z \in$ $D \backslash\left\{\bigcup_{n=1}^{\infty} G_{n}\right\}$ where $G_{n}$ is defined in Lemma 3.2.

PROOF. Using Lemma 3.1, this result can be proved as in [6, Lemma 7]. For completeness we give an outline of a modification of the proof of [6, Lemma 7]. Let

$$
W=\left\{r e^{i t}: 3 / 4<r<1, t \in(-1 / 4,3 / 4)\right\} .
$$

Since $u\left(e^{i t}\right)=1$ when $t \in(-\pi, \pi] \backslash(0,1 / 2)$, we then have that $\lim _{r \uparrow 1} u\left(r^{e i t}\right)=1$ uniformly for $t \in(-\pi, \pi] \backslash(-1 / 8,5 / 8)$. Hence it is sufficient to find a $C_{1}>0$ such that

$$
u(z) \geq C_{1} \quad \forall z \in W \backslash\left\{\bigcup_{n=1}^{\infty} G_{n}\right\} .
$$

For any $0<r<1$ and $\theta \in R$, define $I(\theta, r)=(\theta-3(1-r), \theta+3(1-r))$. Then

$$
\frac{1-r}{(1-r)^{2}+(\theta-t)^{2}} \geq \frac{\chi_{I(\theta, r)}(t)}{10(1-r)} .
$$

Let $\theta \in(-1 / 4,3 / 4)$ and $3 / 4<r<1$. Then $I(\theta, r) \subset(-\pi, \pi]$ and $u\left(e^{i t}\right)=1$ for $t \in I(\theta, r) \backslash U$, where $U=\bigcup_{n=1}^{\infty} I_{n}$. By (3.3) and (3.5),

$$
u\left(r e^{i \theta}\right) \geq \frac{\alpha \cdot m(I(\theta, r) \backslash U)}{10(1-r)} .
$$

Furthermore, assume that $r e^{i \theta} \in W \backslash\left\{\bigcup_{n=1}^{\infty} G_{n}\right\}$. We consider the following two cases.

(i) If $\theta \in(-1 / 4,3 / 4) \backslash U$, then we apply Lemma 3.1 to the case where $x=\theta$ and $a=3(1-r)$ to get

$$
m(I(\theta, r) \backslash U) \geq 3(1-r) / 2 .
$$

By (3.7), it follows that $u\left(r e^{i \theta}\right) \geq 3 \alpha / 20$ in this case. 
(ii) If $\theta \in U$, then there exists $n$ such that $\theta \in I_{n}$. Because $r e^{i \theta} \notin G_{n}, 1-r>\rho_{n}$, the length of $I_{n}$. Since the distance between $\theta$ and $J_{n} \backslash I_{n}$ is less than $\rho_{n}$, we can pick a $\theta^{\prime} \in J_{n} \backslash I_{n}$ such that $\left|\theta-\theta^{\prime}\right|<\rho_{n}<1-r$. Thus

$$
\left(\theta^{\prime}-2(1-r), \theta^{\prime}+2(1-r)\right) \subset I(\theta, r) .
$$

Since $\theta^{\prime} \in \mathbb{R} \backslash U$, apply Lemma 3.1 to the case where $x=\theta^{\prime}$ and $a=2(1-r)$, and the statement follows from (3.6).

It is well known that $C_{\varphi}$ is compact on $L_{a}^{2}(D)$ if and only if

$$
\lim _{|z| \rightarrow 1} \frac{1-|z|}{1-|\varphi(z)|}=0 .
$$

See, for example, $[1,5,7]$. We show that there exists $\varphi$ such that $C_{\varphi}$ is compact but not in $\mathcal{S}_{p}$ for every $1<p<\infty$.

THEOREM 3.4. There exists a composition operator $C_{\varphi}: L_{a}^{2}(D) \rightarrow L_{a}^{2}(D)$ which is not in $\mathcal{S}_{p}$ for any $1<p<\infty$.

PROOF. It is sufficient to construct a compact composition operator $C_{\varphi}$ that does not belong to $\mathcal{S}_{p}$ for $2<p<\infty$. If we can construct $\varphi$ with

$$
\lim _{|z| \rightarrow 1} \frac{1-|z|}{1-|\varphi(z)|}=0 \text { and } \int_{D} \frac{\left(1-|z|^{2}\right)^{p-2}}{\left(1-|\varphi(z)|^{2}\right)^{p}} d A(z)=\infty
$$

for every $2<p<\infty$, we are done. Let

$$
F_{n}=\left\{r e^{i \theta}: \theta \in I_{n}, \rho_{n} / 2<1-r \leq \rho_{n}\right\} \subset G_{n} ;
$$

then $A\left(F_{n}\right) \approx \rho_{n}^{2}$. Since

$$
\begin{aligned}
\int_{D} \frac{(1-|z|)^{p-2}}{(1-|\varphi(z)|)^{p}} d A(z) & \geq \sum_{n=1}^{\infty} \int_{F_{n}} \frac{(1-|z|)^{p-2}}{(1-|\varphi(z)|)^{p}} d A(z) \\
& \succeq \sum_{n=1}^{\infty} \frac{f(n)^{p}}{x(n+1)^{p}}
\end{aligned}
$$

and

$$
\frac{1-|z|}{1-|\varphi(z)|} \preceq \frac{f(n)}{x(n+1)} \quad \text { if } z \in G_{n}
$$

if we let

$$
M=\sum_{n=1}^{\infty} 1 /(n+1)^{2}, \quad f(n)=1 /\left(2 M(n+1)^{2}\right)
$$

and $x(n)=\ln (n+3) f(n)$, then $C_{\varphi}$ is compact and does not belong to $\mathcal{S}_{p}$ for any $1<p<\infty$. 


\section{Acknowledgements}

The authors would like to thank the editor and the referee(s) for their helpful comments and valuable suggestions for improving this paper. The authors are also deeply indebted to Ruhan Zhao for his help with this paper.

\section{References}

[1] C. Cowen and B. MacCluer, Composition Operators on Spaces of Analytic Functions (CRC Press, Boca Raton, FL, 1995).

[2] Z̆ С̆ Ččković and R. Zhao, 'Weighted composition operators on the Bergman space', J. Lond. Math. Soc. 70 (2004), 499-511.

[3] P. Lefévre, D. Li, H. Queffélec and L. Rodríguez-Piazza, 'Some examples of compact composition operators on $H^{2}$, J. Funct. Anal. 255 (2008), 3098-3124.

[4] D. Luecking and K. Zhu, 'Composition operators belonging to the Schatten ideals', Amer. J. Math. 114 (1992), 1127-1145.

[5] J. H. Shapiro, Composition Operators and Classical Function Theory (Springer, Berlin, 1993).

[6] J. Xia, 'On a proposed characterization of Schatten-class composition operators', Proc. Amer. Math. Soc. 131 (2003), 2505-2514.

[7] K. Zhu, Operator Theory in Function Spaces (Marcel Dekker, New York, 1990).

[8] K. Zhu, 'Schatten class composition operators on weighted Bergman spaces of the disk', J. Operator Theory 46 (2001), 173-181.

[9] K. Zhu, 'Schatten class Toeplitz operators on weighted Bergman spaces of the unit ball', New York J. Math. 13 (2007), 299-316.

CHENG YUAN, School of Science, Tianjin University of Technology and Education, Tianjin, PR China

e-mail: yuancheng1984@163.com

ZE-HUA ZHOU, Department of Mathematics, Tianjin University, Tianjin 300072, PR China

e-mail: zehuazhou2003@yahoo.com.cn 